\title{
Spectroscopy of doubly heavy baryons at $\mathrm{LHCb}$
}

\author{
Murdo Thomas Traill ${ }^{* \dagger}$ \\ University of Glasgow \\ E-mail: murdo.thomas.traillecern.ch
}

The LHCb detector is ideally designed for studies in hadron spectroscopy. Its capabilities in this area of physics was firmly demonstrated when $\mathrm{LHCb}$ announced the discovery of the first ever doubly heavy baryon, $\Xi_{c c}^{++}$, in decays of $\Xi_{c c}^{++} \rightarrow \Lambda_{c}^{+} K^{-} \pi^{+} \pi^{+}$in 2017. This doubly charmed baryon was observed as a highly significant structure in the $\Lambda_{c}^{+} K^{-} \pi^{+} \pi^{+}$mass spectrum from proton-proton collision data recorded by the LHCb detector at both $13 \mathrm{TeV}$ and $8 \mathrm{TeV}$ centre-ofmass energies. From the $13 \mathrm{TeV}$ and $8 \mathrm{TeV}$ samples, a yield of $313 \pm 33$ and $113 \pm 21 \Xi_{c c}^{++}$ candidates are measured respectively. The local significances is in excess of $12 \sigma$ in $13 \mathrm{TeV}$ data and above $7 \sigma$ in $8 \mathrm{TeV}$ data. The properties of the peak suggest it is inconsistent with being a strongly decaying state. From the $13 \mathrm{TeV}$ data, the mass is measured to be $3621.40 \pm 0.72$ (stat.) \pm 0.27 (syst.) $\pm 0.14\left(\Lambda_{c}^{+}\right) \mathrm{MeV} / c^{2}$. More analyses in the double heavy baryon sector using $\mathrm{LHCb}$ data are now well advanced. This includes, but is not limited to, searches for the singly charged $\Xi_{c c}^{+}$state and also charmed-beauty $\Xi_{b c}$ baryons.

XVII International Conference on Hadron Spectroscopy and Structure - Hadron2017 25-29 September, 2017

University of Salamanca, Salamanca, Spain

* Speaker.

${ }^{\dagger}$ on behalf of the LHCb collaboration 


\section{Introduction}

The LHCb collaboration have been highly active in hadron spectroscopy ever since the start of the LHC physics program. Among other work, LHCb have contributed significantly to the understanding of $B_{c}^{+}$physics and helped established the existence of some exotic strong states. Following its success in the spectroscopy of hadrons containing a single heavy quark, it was hoped that LHCb would observe a doubly heavy baryon soon. In 2017, the LHCb collaboration announced the discovery of the doubly charmed $\Xi_{c c}^{++}$baryon in data collected in 2012 and 2016 at the LHCb detector [1]. These Proceedings primarily summarize the published work from this discovery analysis but it also discusses future work on baryons containing more than one heavy flavour quark.

The constituent quark model predicts the existence of several hadron states, whose structures are determined by the symmetry properties of their wave-functions. One such prediction is the presence in nature of three weakly decaying ground states that contain two charm valency quarks and have $J^{P}=\frac{1}{2}^{+}$spin-parity assignments. These states are the $\Xi_{c c}^{+}(\mathrm{ccd})$ and $\Xi_{c c}^{++}(\mathrm{ccu})$ baryons, which form an isospin doublet and the $\Omega_{c c}^{+}$(ccs), which forms an isosinglet. Many theoretical models have attempted to calculate the properties of doubly heavy baryons, including their mass, lifetime, production rates and the spectroscopy of their excited states [1]. There had never been any unambiguous experimental evidence for any doubly heavy (or triply heavy) state however. The only previously published evidence of their existence are the observations of the $\Xi_{c c}^{+}$(ccd) baryon claimed by the SELEX collaboration between 2002 and 2004 [2]. They claimed an observation (6.3 $\sigma$ significance) of the $\Xi_{c c}^{+}$state in the $\Lambda_{c}^{+} K^{-} \pi^{+}$final state and followed this up with evidence (4.8 $\sigma$ significance) of the same baryon in the $D^{+} p K^{-}$final state. The combined average mass from these two measurements was $3518.7 \pm 1.7 \mathrm{MeV} / c^{2}$ but the lifetime was unexpectedly short (less than 33 fs at the $90 \%$ confidence level). The production rate was also several orders of magnitude larger than any theoretical models predicts for SELEX's production environment [3]. Many other groups (including LHCb) have searched for SELEX's $\Xi_{c c}^{+}$in the past but none have found evidence for it or any other doubly charmed baryon. This includes the FOCUS collaboration with its studies into doubly charmed baryon production in photon-on-fixed-target collisions [4] as well as the BaBar [5] and Belle [6] groups looking in $e^{+} e^{-}$collisions.

LHCb was designed to be a leader in flavour physics [7] and as such its collects the largest data sets of charmed hadrons in the world. This makes the LHCb experiment an ideal laboratory for the further study of doubly heavy baryons and although LHCb's first search for the $\Xi_{c c}^{+}$state in 2011 [8] (in decays of $\Lambda_{c}^{+} K^{-} \pi^{+}$) failed to find evidence for such a state ${ }^{1}$, the knowledge gained from that analysis ultimately helped towards the discovery of the $\Xi_{c c}^{++}$baryon, a monumental achievement in hadron spectroscopy that should lead to further success in the study of doubly heavy baryons.

\section{Discovery of $\Xi_{c c}^{++}$baryon}

The ground states of doubly charmed baryons have always been the main focus of doubly heavy baryon searches at $\mathrm{LHCb}$; mostly because their production cross-sections are expected to be significantly higher than baryons containing both bottom and charm quarks. LHCb's original focus was the $\Xi_{c c}^{+}$but prompted by the theoretical community, LHCb switched instead to looking

\footnotetext{
${ }^{1}$ The sensitivity of the analysis was found to be limited by design choices in the trigger and selections.
} 

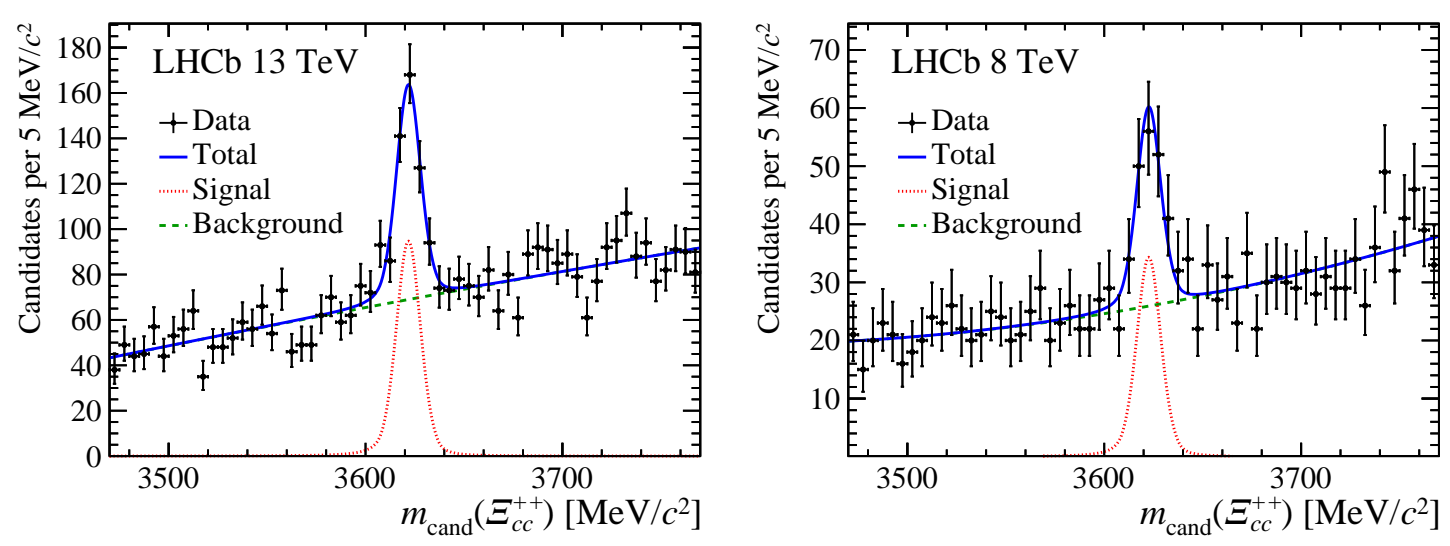

Figure 1: Distribution of the $\Lambda_{c}^{+} K^{-} \pi^{+} \pi^{+}$mass spectrum for candidates passing the analysis selection for the $13 \mathrm{TeV}$ (left) and $8 \mathrm{TeV}$ (right) LHCb data set. The fits near the peak region are shown as well.

for its isospin partner, $\Xi_{c c}^{++}$, at the start of 2016. The reason was based on the predicted larger lifetime of the $\Xi_{c c}^{++}$state compared to its singly charged isospin partner. It meant triggering and selections should have been more efficient at removing backgrounds created at the $p p$ interaction point where signals of $\Xi_{c c}^{++} \rightarrow \Lambda_{c}^{+} K^{-} \pi^{+} \pi^{+}$should also be produced. The search channel chosen was $\Xi_{c c}^{++} \rightarrow \Lambda_{c}^{+} K^{-} \pi^{+} \pi^{+}$as it was expected to have a larger branching fraction compared to any other hadronic mode under consideration [9].

In 2017, LHCb announced they had found a prominent and narrow structure in the $\Lambda_{c}^{+} K^{-} \pi^{+} \pi^{+}$ mass spectrum resulting from $p p$ collisions produced by the Large Hadron Collider. The structure was found to be consistent with the observation of a doubly charmed $\Xi_{c c}^{++}$baryon decaying to a $\Lambda_{c}^{+} K^{-} \pi^{+} \pi^{+}$final state. The data sets for the discovery came from collisions at a center-of-mass (CoM) energy of $13 \mathrm{TeV}$ and $8 \mathrm{TeV}$ corresponding to an integrated luminosity of $1.7 \mathrm{fb}^{-1}$ and $2.0 \mathrm{fb}^{-1}$ respectively. The two data sets are analysed independently of one another and in both cases a observation of the $\Xi_{c c}^{++}$is made. However, because of the higher yield and greater statistical significance in the $13 \mathrm{TeV}$ sample, the analysis of that data set is used as the primary result in the LHCb publication. The analysis of the $8 \mathrm{TeV}$ data set on the other hand is mostly placed in the supplementary material which can be found on CDS [1].

The $\Xi_{c c}^{++}$candidates were reconstructed from the decay chain $\Xi_{c c}^{++} \rightarrow \Lambda_{c}^{+}\left(\rightarrow p K^{-} \pi^{+}\right) K^{-} \pi^{+} \pi^{+}$ in both data samples and are required to pass selections based on various quantities including reconstruction quality metrics, particle identification scores and topological and kinematic information. A multivariate discriminant, trained to separate simulated $\Xi_{c c}^{++} \rightarrow \Lambda_{c}^{+} K^{-} \pi^{+} \pi^{+}$decays from background represented by wrong-sign (WS) data with an unphysical combination of charged tracks $\left(\Lambda_{c}^{+} K^{-} \pi^{+} \pi^{-}\right)$, is also used to prepare the final samples used in the mass measurement. The MVA selectors make use of quality, topological and dynamical information from the decays.

\subsection{Analysis details}

The $13 \mathrm{TeV}$ data used in LHCb's primary analysis was collected throughout 2016 using a dedicated exclusive trigger which a high efficiency. In this sample, $\Xi_{c c}^{++}$candidates are produced by LHCb's Turbo processing which reconstructs the entire decay online in the software trigger 
[10]. Since there is no further event reconstruction, the storage required to keep these Turbo events is greatly reduced; an important feature for analyses involving large data sets such is the case for the intermediate Cabibbo-favoured $\Lambda_{c}^{+} \rightarrow p K^{-} \pi^{+}$decay in this $\Xi_{c c}^{++} \rightarrow \Lambda_{c}^{+} K^{-} \pi^{+} \pi^{+}$channel.

After the final selection is applied to the $\Xi_{c c}^{++}$candidates in data, the $\Lambda_{c}^{+} K^{-} \pi^{+} \pi^{+}$mass spectrum in the range $[3300,3800] \mathrm{MeV} / c^{2}$ shows a significant structure around $3600 \mathrm{MeV} / \mathrm{c}^{2}$. However in the spectrum of $\left[p K^{-} \pi^{+}\right]_{S B} K^{-} \pi^{+} \pi^{+}$candidates (where $\left[p K^{-} \pi^{+}\right]_{S B}$ denotes combinations taken from the sidebands of the $\Lambda_{c}^{+}$mass distribution) no matching peak is observed. Similarly once the same analysis cuts are applied to the WS $\left(\Lambda_{c}^{+} K^{-} \pi^{+} \pi^{-}\right)$mass spectrum no peaking structures are observed in the region $[3300,3800] \mathrm{MeV} / c^{2}$ but the distribution is similar otherwise.

The left plot in Figure 1 shows the mass distribution in data near this narrow peak. The mass variable that is plotted is $m_{\text {cand }}\left(\Xi_{c c}^{++}\right)$. This is the mass difference between the combined mass of the final state particles, $\mathrm{m}\left(\Lambda_{c}^{+} K^{-} \pi^{+} \pi^{+}\right)$and the reconstructed $\Lambda_{c}^{+}$mass, $m_{\text {cand }}\left(\Lambda_{c}^{+}\right)$, corrected by the known value of the $\Lambda_{c}^{+}$mass, $m_{P D G}\left(\Lambda_{c}^{+}\right)=2286.46 \pm 0.14 \mathrm{MeV} / c^{2}[11]$;

$$
m_{\text {cand }}\left(\Xi_{c c}^{++}\right) \equiv m\left(\Lambda_{c}^{+} K^{-} \pi^{+} \pi^{+}\right)-m_{\text {cand }}\left(\Lambda_{c}^{+}\right)+m_{P D G}\left(\Lambda_{c}^{+}\right)
$$

The left plot in Figure 1 also shows the result of the fit function overlaid on the mass distribution. The extracted yield of $\Xi_{c c}^{++}$candidates in $13 \mathrm{TeV}$ data is measured to be $313 \pm 33$ events. This corresponds to a local significance greater than $12 \sigma$ when evaluated with a likelihood ratio test. The width of the peak $\left(6.6 \pm 0.8 \mathrm{MeV}^{2}\right)$ is consistent with the expected detector resolution and the mass difference between the $\Xi_{c c}^{++}$state and the $\Lambda_{c}^{+}$baryon is found to be $1334.94 \pm 0.72$ (stat.) \pm 0.27 (syst.) $\mathrm{MeV} / \mathrm{c}^{2}$ following corrections of known detector and reconstruction effects. The standalone mass measurement of the $\Xi_{c c}^{++}$state is observed to be $3621.40 \pm 0.72$ (stat.) \pm 0.27 (syst.) $\pm 0.14\left(\Lambda_{c}^{+}\right) \mathrm{MeV} / c^{2}$ where the final uncertainty is that from the world-average $\Lambda_{c}^{+}$mass measurement. The main contributions to the systematic uncertainty are the uncertainty of the momentum scale correction of the reconstruction and the uncertainty of the correction to account for correlations between the reconstructed mass of the candidates and the selection variables.

\subsection{Complementary observation}

To verify the discovery, $\mathrm{LHCb}$ also studied data collected at the $8 \mathrm{TeV}$ CoM energy in parallel to primary $13 \mathrm{TeV}$ analysis ${ }^{2}$. This data was recorded with a different trigger and data processing setup than the $13 \mathrm{TeV}$ data sample [12]. Events for this $8 \mathrm{TeV}$ analysis were chosen by a software trigger by selecting $\Lambda_{c}^{+} \rightarrow p K^{-} \pi^{+}$decays, similar to the $13 \mathrm{TeV}$ case. However, instead of directly analysing the $\Lambda_{c}^{+}$candidates reconstructed in the trigger, a further reconstruction is added to improve the precision. If the $\Lambda_{c}^{+}$candidates from this additional reconstruction are matched to the corresponding trigger candidates with a certain level of confidence, then these $\Lambda_{c}^{+}$candidates are used to create the $\Lambda_{c}^{+} K^{-} \pi^{+} \pi^{+}$and WS $\Lambda_{c}^{+} K^{-} \pi^{+} \pi^{-}$mass combinations.

The selection is designed in a similar manner to the $13 \mathrm{TeV}$ analysis, with loose rectangular cuts on the same quantities applied and the same multivariate selector used. The differences that arise in comparison to the $13 \mathrm{TeV}$ analysis are in the cut values themselves which need changed

\footnotetext{
${ }^{2}$ The analysis of the $8 \mathrm{TeV}$ data set is described in detail in the supplemental material released with the publication of the primary analysis [1].
} 

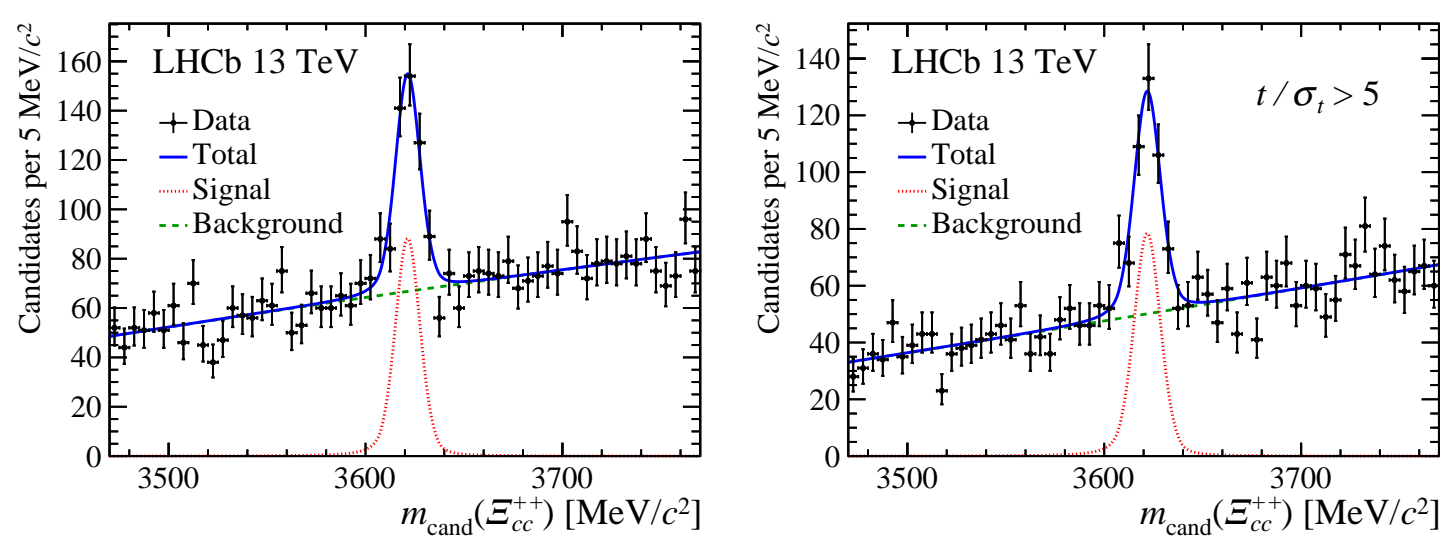

Figure 2: Distribution of the $\Lambda_{c}^{+} K^{-} \pi^{+} \pi^{+}$mass spectrum in the $13 \mathrm{TeV}$ data for candidates passing a selection based only on rectangular cuts (left) and for the candidates which have a reconstructed lifetime five times greater than its decay time resolution.

to reflect the differences in the trigger, reconstruction and optimal working point for the expected $\Xi_{c c}^{++}$production kinematics in $8 \mathrm{TeV} p p$ collisions.

Despite these differences, a clear peak is once again observed in the $\Lambda_{c}^{+} K^{-} \pi^{+} \pi^{+}$mass spectrum from the $8 \mathrm{TeV}$ data sample with similarly no structure being seen in the mass spectra of either the WS $\Lambda_{c}^{+} K^{-} \pi^{+} \pi^{-}$candidates or the $\left[p K^{-} \pi^{+}\right]_{S B} K^{-} \pi^{+} \pi^{+}$candidates from the $\Lambda_{c}^{+}$mass sidebands. The right plot of Figure 1 shows the $m_{\text {cand }}\left(\Xi_{c c}^{++}\right)$distribution for the final $8 \mathrm{TeV}$ sample passing the selection. The fit to determine the peak position and yield is also shown. With the $8 \mathrm{TeV}$ data, the local significance of the peak is slightly less than that found in the $13 \mathrm{TeV}$ sample but importantly the peak is found to be in the same place. The difference between the peaks in the two independent data sets is in fact only $0.8 \pm 1.4 \mathrm{MeV} / c^{2}$ (the uncertainty only includes the statistical uncertainties from the fits). The width of the $8 \mathrm{TeV}$ peak $\left(6.6 \pm 1.4 \mathrm{MeV} / \mathrm{c}^{2}\right)$ is again consistent with the LHCb's expected detector resolution and in terms of yield, $113 \pm 21 \Xi_{c c}^{++}$signal decays are found from the resulting fit. When these results are combined with the $13 \mathrm{TeV}$ sample, the total yield of $\Xi_{c c}^{++} \rightarrow \Lambda_{c}^{+} K^{-} \pi^{+} \pi^{+}$decays is $426 \pm 39$ events. Giving the signal peaks in both the $13 \mathrm{TeV}$ and $8 \mathrm{TeV}$ samples (which were analysed independently of one another) show very similar properties, LHCb have complete confidence in its $\Xi_{c c}^{++}$discovery claim.

\subsection{Cross-checks}

Further cross-checks were performed to test the robustness of the $13 \mathrm{TeV}$ and $8 \mathrm{TeV}$ peaks. The threshold value of the MVA selector was varied resulting in no sizeable change on the signal significance, the efficiency of the MVA selector with respect to the $\Xi_{c c}^{++}$invariant mass showed no noticeable biasing effects and also LHCb looked if having multiple $\Xi_{c c}^{++}$candidates in the same event would cause artificial structures to appear but this was not the case. Cut values on particle identification scores of the final state tracks were additionally varied with no peak emerging in the WS combinations but the peak did remain in the $\Lambda_{c}^{+} K^{-} \pi^{+} \pi^{+}$mass spectrum.

The absence of a corresponding feature in the WS $\Lambda_{c}^{+} K^{-} \pi^{-} \pi^{+}$mass spectrum also demonstrates that the $\Xi_{c c}^{++}$peak is not the result of the multivariate discriminant causing some unknown 
biasing. When LHCb removed the multivariate from the selection and replaced it with a set of rectangular cuts based on quality, topological, dynamic and particle identification variables of the $\Xi_{c c}^{++} \rightarrow \Lambda_{c}^{+} K^{-} \pi^{+} \pi^{+}$decays, the peaks remains stable, as can be seen in the left plot of Figure 2. The local statistical significance stays in the excess of $12 \sigma$ as well. The same plot for the $8 \mathrm{TeV}$ data can be viewed in the supplementary material of the publication on CDS [1].

\subsection{Properties of new state}

For this analysis, it was important for LHCb to identify the basic properties of the signal peak found in the $\Lambda_{c}^{+} K^{-} \pi^{+} \pi^{+}$mass spectrum. A mass measurement was completed but the lifetime of $\Xi_{c c}^{++}$state was not determined. Instead a separate analysis is being performed to make this measurement which will be released as part of another publication. However, this analysis does offer preliminary evidence that the state is likely to be a weakly decaying state.

Firstly, the shape of $13 \mathrm{TeV}$ and $8 \mathrm{TeV}$ peaking structures are not well represented by a BreitWigner function that is typical of strong resonances. Secondly, the peaking structure persists after requiring that the reconstructed lifetime of the $\Xi_{c c}^{++}$candidates be greater than five times its estimated resolution, $\sigma_{\tau}$. The right plot of Figure 2 shows the $\mathrm{m}\left(\Xi_{c c}^{++}\right)$distribution for this subset of the $13 \mathrm{TeV}$ data set with $\tau\left(\Xi_{c c}^{++}\right)>5 \sigma_{\tau}$. The signal peak maintains a local significance greater than $12 \sigma$ in the $13 \mathrm{TeV}$ data, while the $8 \mathrm{TeV}$ peak still has a local significance exceeding $7 \sigma$. The fact that the peak remains after placing large displacement cuts indicates that the $\Xi_{c c}^{++}$baryon has a non-negligible lifetime and that it is easily resolvable by the LHCb detector.

\section{Future prospects with doubly heavy baryons}

Since the $\Xi_{c c}^{++}$discovery, $\mathrm{LHCb}$ are continuing to be work on doubly heavy baryon studies with an ever increasing number of analysts involved. The prime focus for $\Xi_{c c}^{++}$related work will be measuring its lifetime and production cross-section. Additional decay modes of the $\Xi_{c c}^{++}$are also being studied to verify if signal can be found in alternative final states. Branching fraction measurements can then also be made relative to the $\Lambda_{c}^{+} K^{-} \pi^{+} \pi^{+}$channel. Further in the future, constraints will be made on the spin and parity of the LHCb's $\Xi_{c c}^{++}$state and CP violation studies in this system could also be conducted once larger data sets are available.

The main objective will be finding the $\Xi_{c c}^{+}$baryon such that direct comparisons can be made to SELEX's $\Xi_{c c}^{+}$state. Work is currently ongoing on this search, with LHCb now able to draw on larger data sets and improvements in triggering and selections for this type of search.

In terms of other doubly heavy systems, searches for $\Omega_{c c}^{++}$maybe also possible in the near future. The lifetime for this state is expected to be similar to the lifetimes predicted for $\Xi_{c c}^{+}$state. The most promising search channels for this state have several final state tracks, more so than any of the current $\Xi_{c c}$ search channels. Therefore, reconstruction efficiency is expected to be low. Searches for the $\Xi_{b c}, \Omega_{b c}$ and $\Xi_{b b}$ baryons are also under development. There is not expected to be one obvious discovery mode for these states so searches for them will be done as a multi-channel analysis. The cross-sections and branching fractions for these particles are also expected to be much smaller than that of doubly charmed baryons [13]. 


\section{Discussion and conclusion}

LHCb's observation of the $\Xi_{c c}^{++}$state represents the first ever published work on this baryon. The SELEX group have shown unpublished work on the $\Xi_{c c}^{++}$baryon at conferences in the past but no peak matching LHCb's newly discovered state exists in their $\Lambda_{c}^{+} K^{-} \pi^{+} \pi^{+}$mass distribution [14]. Furthermore, the published SELEX $\Xi_{c c}^{+}$state has a mass $\left(103 \pm 2 \mathrm{MeV} / c^{2}\right)$ smaller than LHCb's $\Xi_{c c}^{++}$state. The two states are clearly not compatible with being an isodoublet [15] and also cannot be an excitation of the other since both groups claim they see a weakly decaying state.

In conclusion, the mass of LHCb's $\Xi_{c c}^{++}$state is measured to be $3621.40 \pm 0.72$ (stat.) \pm 0.27 (syst.) $\pm 0.14\left(\Lambda_{c}^{+}\right) \mathrm{MeV} / c^{2}$ and this was established having reconstructed $426 \pm 39$ decays of $\Xi_{c c}^{++} \rightarrow$ $\Lambda_{c}^{+} K^{-} \pi^{+} \pi^{+}$in the LHCb detector. The state is consistent with a particle that travels a significant distance before decaying but is inconsistent with being the isospin partner of SELEX's $\Xi_{c c}^{+}$state. Measurements of the lifetime and production rate of this newly discovered particle are in progress, as are searches for $\Xi_{c c}^{++}$decaying to additional decay modes. Searches for the $\Xi_{c c}^{+}$and $\Omega_{c c}^{+}$baryons and measurements of their properties have also begun.

The discovery of the $\Xi_{c c}^{++}$baryon represents the beginning of an exciting time of doubly heavy baryon research at LHCb. The aim for the collaboration going forward will now be to build an accurate and concise picture of doubly heavy baryons as a whole.

\section{References}

[1] LHCB collaboration, R. Aaij et al., Observation of the doubly charmed baryon $\Xi_{c c}^{++}$, Phys. Rev. Lett. 119 (2017) 112001, [1707.01621].

[2] SELEX collaboration, M. Mattson et al., First observation of the doubly charmed baryon Xi+(cc), Phys. Rev. Lett. 89 (2002) 112001, [hep-ex/0208014].

[3] V. V. Kiselev and A. K. Likhoded, Baryons with two heavy quarks, Phys. Usp. 45 (2002) 455-506, [hep-ph/0103169].

[4] S. P. Ratti, J. Link, M. Reyes, P. Yager, J. Anjos, I. Bediaga et al., New results on c-baryons and a search for cc-baryons in focus, Nuclear Physics B - Proceedings Supplements 115 (2003) 33 - 36.

[5] BABAR collaboration, B. Aubert et al., Search for doubly charmed baryons Xi(cc)+ and Xi(cc)++ in BABAR, Phys. Rev. D74 (2006) 011103, [hep-ex/ 0605075 ].

[6] BELle collaboration, R. Chistov et al., Observation of new states decaying into Lambda(c)+ K- pi+ and Lambda(c)+KO(S) pi-, Phys. Rev. Lett. 97 (2006) 162001, [hep-ex/ 0606051 ].

[7] LHCB collaboration, R. Aaij et al., LHCb Detector Performance, Int. J. Mod. Phys. A30 (2015) 1530022, [1412.6352].

[8] LHCB collaboration, R. Aaij et al., Search for the doubly charmed baryon $\Xi_{c c}^{+}$, JHEP 12 (2013) 090, [1310.2538].

[9] F.-S. Yu, H.-Y. Jiang, R.-H. Li, C.-D. LÃij, W. Wang and Z.-X. Zhao, Discovery Potentials of Doubly Charmed Baryons, 1703.09086.

[10] R. Aaij, S. Amato, L. Anderlini, S. Benson, M. Cattaneo, M. Clemencic et al., Tesla: An application for real-time data analysis in high energy physics, Computer Physics Communications 208 (2016) 35 -42 . 
[11] Particle Data Group collaboration, C. Patrignani et al., Review of Particle Physics, Chin. Phys. C40 (2016) 100001.

[12] R. Aaij, J. Albrecht, F. Alessio, S. Amato, E. Aslanides, I. Belyaev et al., The lhcb trigger and its performance in 2011, Journal of Instrumentation 8 (2013) P04022.

[13] J.-W. Zhang, X.-G. Wu, T. Zhong, Y. Yu and Z.-Y. Fang, Hadronic Production of the Doubly Heavy Baryon $\Xi_{b c}$ at LHC, Phys. Rev. D83 (2011) 034026, [1101.1130].

[14] SELEX collaboration, J. Engelfried, SELEX: Recent Progress in the Analysis of Charm-Strange and Double-Charm Baryons, eConf C0610161 (2006) 003, [hep-ex/ 0702001 ].

[15] M. Karliner and J. L. Rosner, Isospin splittings in baryons with two heavy quarks, Phys. Rev. D96 (2017) 033004, [1706.06961]. 